\title{
Indomethacin Sodium
}

National Cancer Institute

\section{Source}

National Cancer Institute. Indomethacin Sodium. NCI Thesaurus. Code C47999.

The sodium salt of indomethacin, a methylated indole derivative with anti-inflammatory, analgesic-antipyretic and tocolytic effects. Indomethacin is a non-selective, reversible, and competitive inhibitor of cyclooxygenases 1 and 2, thereby blocking the conversion of arachidonic acid into prostaglandin precursors. Consequently, prostaglandin synthesis is decreased, and prostag landin-mediated activities are prevented, including pain, inflammation, fever and uterine contraction. 